\title{
ESE guidelines, why and how
}

\author{
Olaf M Dekkers ${ }^{1,2,3}$, Pia Burman ${ }^{4}$ and On behalf of the ESE Clinical Committee \\ ${ }^{1}$ Department of Medicine, Division of Endocrinology, and ${ }^{2}$ Department of Clinical Epidemiology, Leiden University \\ Medical Center, Leiden, Netherlands, ${ }^{3}$ Department of Clinical Epidemiology, Aarhus University Hospital, Aarhus, \\ Denmark and ${ }^{4}$ Department of Endocrinology, Skane University Hospital, Malmö, Sweden
}

Correspondence should be addressed to O M Dekkers

Email

o.m.dekkers@lumc.nl
In the present issue of the European Journal of Endocrinology, the first European Society of Endocrinology (ESE) Guideline is being published. This publication will mark the starting point of a tradition in high quality guidelines. In 2013, ESE decided to develop evidence-based guidelines in endocrinology aiming to improve and harmonize patient care across Europe. The topics will ideally address areas not previously covered by other influential endocrine societies/specialty organizations, or areas where available recommendations are not applicable to the European standard of care. To identify potentially relevant guideline topics, the ESE Clinical Committee completed a review of more than 150 guidelines, position papers, and consensus statements issued by a number of endocrine societies, specialty organizations and expert groups in Europe and the USA from 2005 to 2013. The main aim of this review was to gain an overview of topics well covered in existing guidelines. Subsequently, two priority areas were selected for guideline development by the ESE: management of chronic hypoparathyroidism and management of adrenal incidentalomas. Also, a European working group was formed, dedicated to summarizing evidence on long-term management after surgery for pheochromocytoma systematically. It was also decided to develop a guideline for this topic as well.

Each guideline is guided by two chairs, a content expert and a methodologist. As a second step, a team was built consisting of clinical experts, and if needed from different specialties. The first draft of the guideline would be open for comments from ESE members and all guidelines will be presented at the European Congress of Endocrinology, preferably before final publication. In addition, patient information leaflets will be developed in collaboration with patient support groups.

In an ideal setting, guidelines are based on evidence from randomized trials and accompanied by strong recommendations. Unfortunately, most areas in endocrinology (with exceptions of diabetes and osteoporosis) are not well covered by randomized trials, and available evidence is often weak, up to being non-existent. As can be seen in the hypoparathyroidism guideline (1), none of the recommendations is supported by strong evidence. These limitations in available evidence challenges a guideline panel. The use of a systematic and widely adopted methodological approach (the GRADE system for guideline development (2)) is not a 'magic bullet', able to transform the quality of evidence to a higher level. The obvious alternative option, to abstain from recommendations in the absence of evidence is a no-go, for the very simple reason that one cannot abstain from treatment decisions for patients, even in the absence of evidence. However, it is important to emphasize a lack of solid evidence in the guideline, to acknowledge the larger role of expert opinions and to resist strong recommendations in these cases. As such, it is acknowledged that a good practice statement should go without formal assessment of the evidence, as the lack of apparent evidence might wrongly suggest the lack of a good rationale for clinical practice (3). That a diagnosis of hypoparathyroidism should be considered in patients with a low serum calcium level is an example of good clinical practice not in need of further evidence.

Many publications end by stating that, 'more research is needed'. Such a statement is meaningless, unless specified in terms of research questions and methodological approaches. The ESE guidelines will be accompanied by a systematic summary of the literature which may point towards important gaps in clinical knowledge. Therefore, the guidelines provide an opportunity to identify specified suggestions for future research. All ESE guidelines will incorporate a paragraph with these suggestions, which hopefully initiate collaborative efforts to study clinically relevant questions.

What is the road ahead? In the next years, guideline development will be a prominent task of the ESE Clinical Committee. Taking resources and person-time into

Published by Bioscientifica Ltd. 
account, a scenario with publication of one ESE guideline every year seems realistic. Suggestions for new guidelines are welcomed, as long as the topic fits the formulated aim. A guideline should not be a mere repetition of other guidelines, and the proof of this is its clinical relevance for clinicians and patients.

\section{Declaration of interest}

The authors declare that there is no conflict of interest that could be perceived as prejudicing the impartiality of the editorial.

\section{Funding}

This editorial did not receive any specific grant from any funding agency in the public, commercial or not-for-profit sector.

\section{References}

1 Bollerslev J, Rejnmark L, Marcocci C, Shoback DM, Sitges-Serra A, van Biesen W \& Dekkers OM. European Society of Endocrinology Clinical Guideline: Treatment of Chronic Hypoparathyroidism in Adults. European Journal of Endocrinology 2015173 G1-G20. (doi:10.1530/EJE-15-0628)

2 Guyatt G, Oxman AD, Akl EA, Kunz R, Vist G, Brozek J, Norris S, Falck-Ytter Y, Glasziou P, DeBeer H et al. GRADE guidelines: 1. Introduction-GRADE evidence profiles and summary of findings tables. Journal of Clinical Epidemiology 201164 383-394. (doi:10.1016/ j.jclinepi.2010.04.026)

3 Guyatt GH, Schünemann HJ, Djulbegovic B \& Akl EA. Guideline panels should not GRADE good practice statements. Journal of Clinical Epidemiology 201568 597-600. (doi:10.1016/j.jclinepi.2014. 12.011)

Received 24 June 2015

Accepted 25 June 2015 